\title{
DIAGNOSTIC OVERVIEW PRESENTED AT DRY EYE UNIVERSITY
}

\author{
Jerry Robben, OD
}

Chief Optometrist and Director of Clinical Research at Bowden Eye \& Associates in Jacksonville, FL. Adjunct Clinical Professor at Midwestern University, College of Optometry in Glendale, AZ.

\section{Corresponding Author: jrobben@bowdeneye.com}

Published: April 16. 2020.

We understand more about dry eye disease than ever before. This expanded knowledge has allowed innovators to identify key components of dry eye disease and develop ways to actually measure them. This understanding and now the application of these measurable components is allowing eye care providers greater knowledge in understanding their patient's dry eye disease status. Being able to obtain these dry eye metrics is helping to diagnose patients earlier by allowing us to see functional, environmental and structural changes in the dry eye patient earlier in the disease state. These metrics can help us to better customize our treatment plans, by being able to identify the exact component or components of the lacrimal functional unit that needs attention. With these, we can confirm if our treatments are working or not and to help validate for ourselves and our patients that our proposed treatments are sound. And we are better able to track improvement versus stability versus progression by using these metrics consistently over time.

Long gone are the days of relying only on a Schirmer's test and ocular surface staining. Both of these are still valid and appropriate. I rely on fluorescein and lissamine green on nearly every dry eye evaluation., but our clinic does not routinely perform Schirmer's test anymore. I don't mean that as a knock on it. We just don't feel the need to devote the time now that we have all of these other diagnostics available.
This section outlines the dry eye diagnostics that we present in great detail at Dry Eye University. We allow each of the representatives for these to address our audience, and our attendees are encouraged to get hands-on experience with each of these tests during the program. Our list is not complete. There are many other diagnostics in the dry eye space that are not listed here. Our list includes the only tests that come to DEU. At DEU we only allow treatment, diagnostics, or practice management aids to be represented if we have experience with the product in our practice, Bowden Eye \& Associates in Jacksonville, FL. We have made this requirement so that we can give our attendees actual real-world experience on our content and not just talking points provided by each company.

What I call "The Dry Eye Explosion" occurred, for our practice, with our introduction and utilization of the Tear Science Lipiview and Lipiflow system, (now part of Johnson \& Johnson Vision) along with the Tear Lap osmolarity testing and Inflammadry mmp-9 testing that is now owned by Quidel. We quickly learned how to integrate these diagnostics together to make them all make sense to us. This allowed us to progress greatly in our ability to identify patients earlier in the dry eye disease continuum and better educate them about their condition. This information provided by these diagnostics also helped us to guide treatment 
plans better, assess control vs progression and just take better care of our patients.

Tear Osmolarity testing with the TearLab unit was, and still is, integral in our clinics. Tear osmolarity is simply the measurement of salts in the tear solution.
Studies suggest that when the salt content of the tears is high (hyperosmolarity) then the ocular surface is damaged. In a dry eye patient, osmolarity will be elevated and variable with possible large swings in readings.

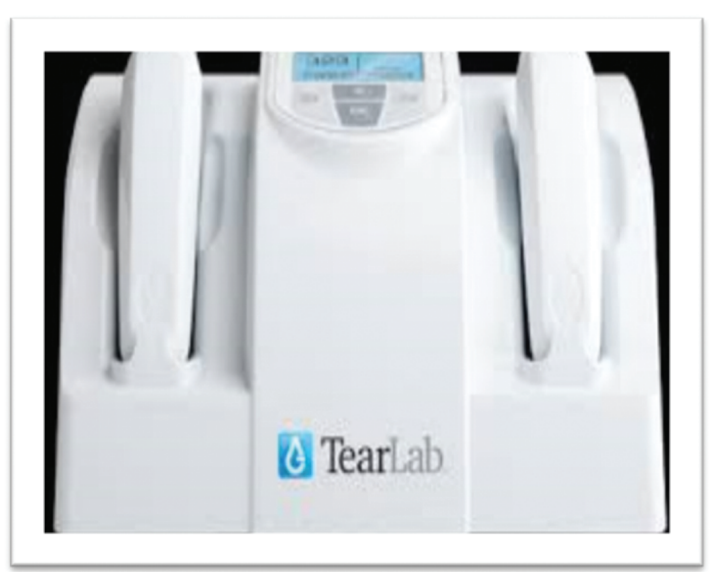

These large swings indicated that the ocular surface is not at homeostasis and implies damage and the presence of dry eye disease. We obtain TearLab testing at every medical visit for a dry eye patient. If dry eye is not formally diagnosed, we will obtain TearLab yearly or if new dry eye symptoms are reported. With osmolarity, the more data points obtained can help start to lay our trends of control vs stability

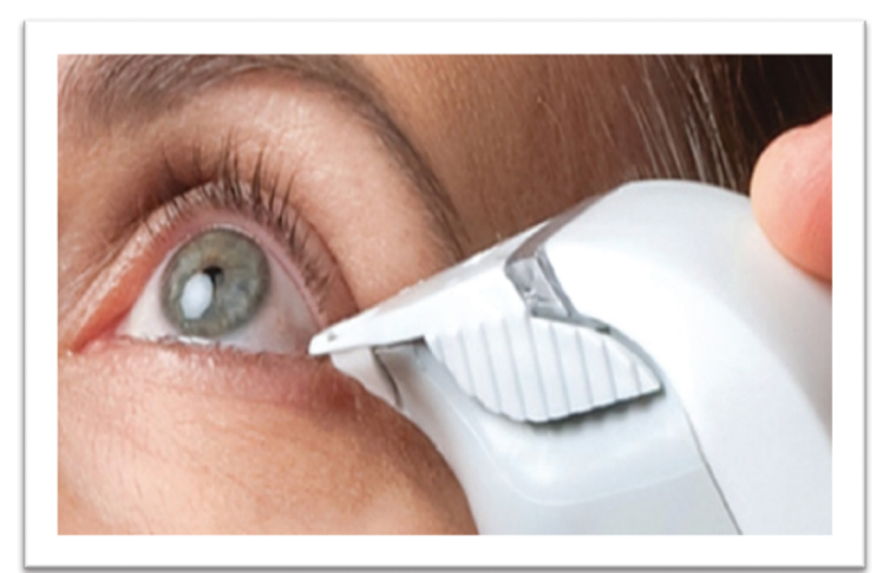

vs progression. The best way to use osmolarity is over the long term. We recommend recording all dry eye diagnostics in a "dry eye worksheet" similar to recording IOP measurements over time to spot trends over time. Abnormal osmolarity can be categorized into severity ranges as shown below. Variable readings between the two eyes with values greater than $8 \mathrm{mOsm} / \mathrm{L}$ also indicated abnormal results.

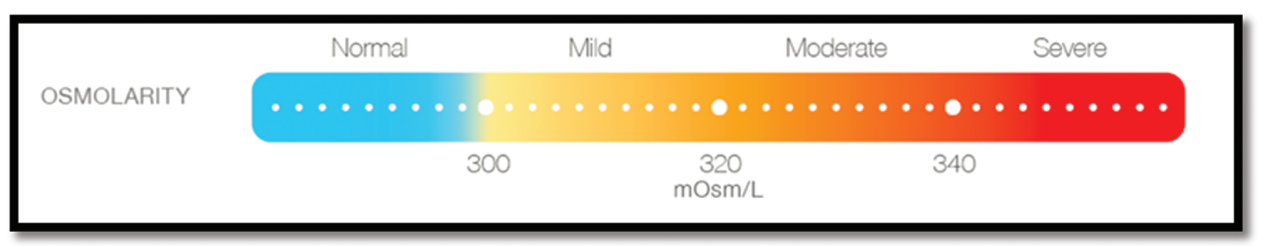

InflammaDry testing has also enhanced our clinics. This test is assessing for elevated levels of matrix metalloproteinase 9 (MMP-9) which is a known inflammatory marker, a cytokine, which is produced by inflamed and or damaged epithelial cells. We are testing for the presence of this marker on the ocular surface, if positive we can infer that the patient is currently experiencing stress to the ocular surface and therefore is not controlled, even with no symptoms and/or signs present. These patients likely require more aggressive dry eye treatments, similar to a glaucoma patient whose current IOP management is not providing enough IOP control. Similar to TearLab, we obtain this test on every new medical patient who has dry eye symptoms or is suspected of dry eye. The technician performs this test just after the tear lab test. If positive we will initiate appropriate anti-inflammatory treatments and then follow up in a short time (usually in 4-6 weeks). At follow up we will repeat this test, if still positive we continue our efforts to gain control, if negative we will not repeat this test until the next yearly exam, or unless new symptoms arise. 


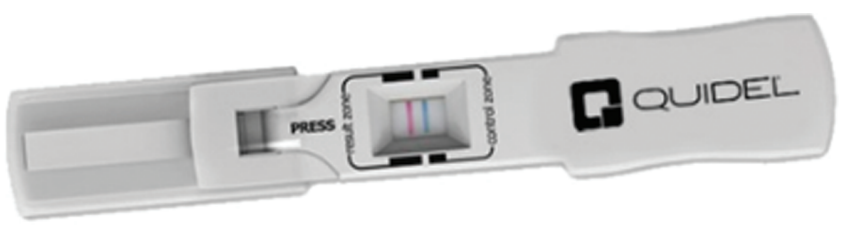

The TearScience, now part of Johnson \& Johnson Vision, Lipiview system was a major catalyst in our early dry eye clinic. This system provided Lipid Layer Thickness (LLT) analysis using interferometry as well as Partial Blink Analysis. Since then the system has been updated to the LipiView II system that provides the LLT and Partial Blink Analysis along with meibography using their advanced Dual Mode Imaging system to provide very high resolution of the meibomian glands. The Lipiview II is a cornerstone in our offices. We have on in multiple locations. We use the LLT/Partial Blink and meibography as a baseline for all dry eye evaluations and then we will repeat the LLT/Partial blink regularly, especially after dry eye interventions. Meibography is usually repeated at least yearly.

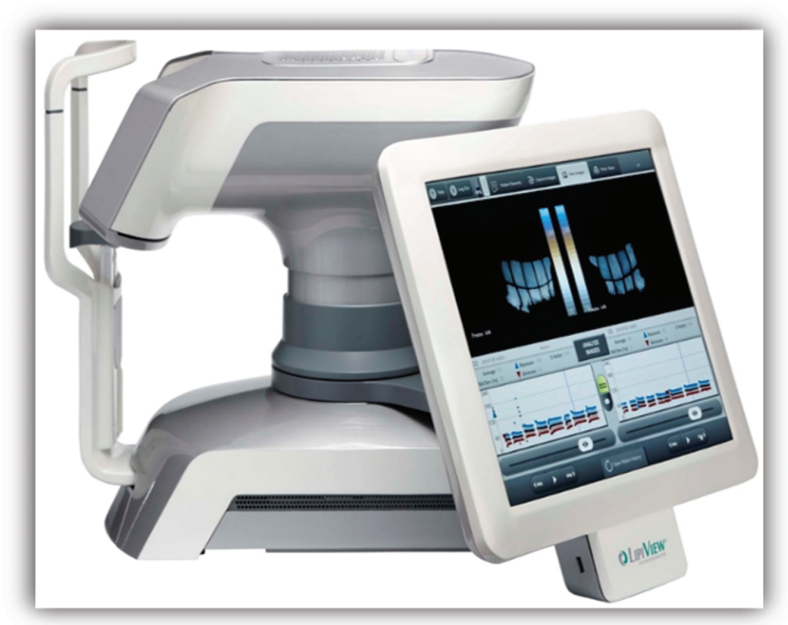

For offices that have less space available, the LipiScan has been developed with a smaller footprint compared to the LipiView II. The LipiScan provide the same DMI Meibography images but does not have the LLT/Partial Blink capability. We use this in some of our smaller locations.
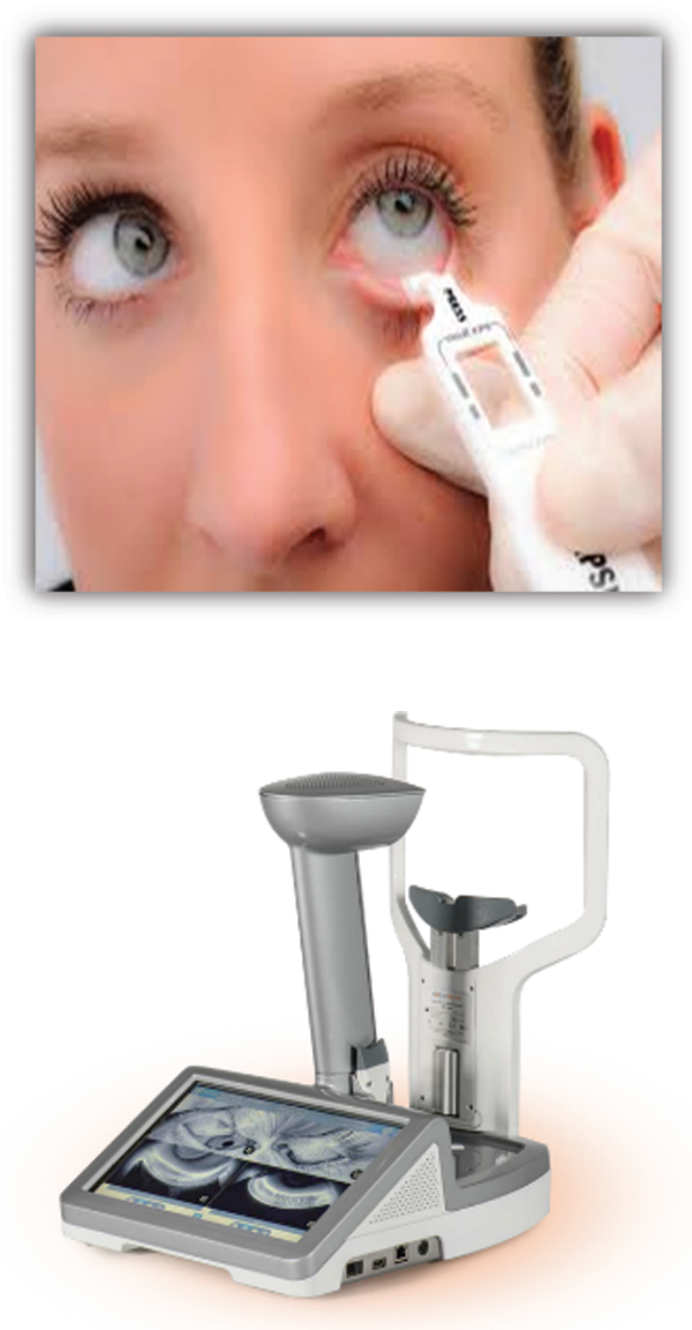

There are other diagnostic platforms that have similar capabilities as the Lipiview system. One we have experience with is the Oculus Keratograph 5M. This device provides multiple dry eye assessments in one device including meibography, Non Invasivis Tear Break Up Time (NIKBUT), Tear Film Analysis, Tear Meniscus Measurement, Ocular Hyperemia Analysis, Anterior Segment Photos and Corneal Topography. The $5 \mathrm{M}$ also includes software that provides the Crystal TEAR Report that provides the patients diagnostic information in one place that can be customized to your desired specifications. This allows easy understanding for the physician and the patient to digest this information. 


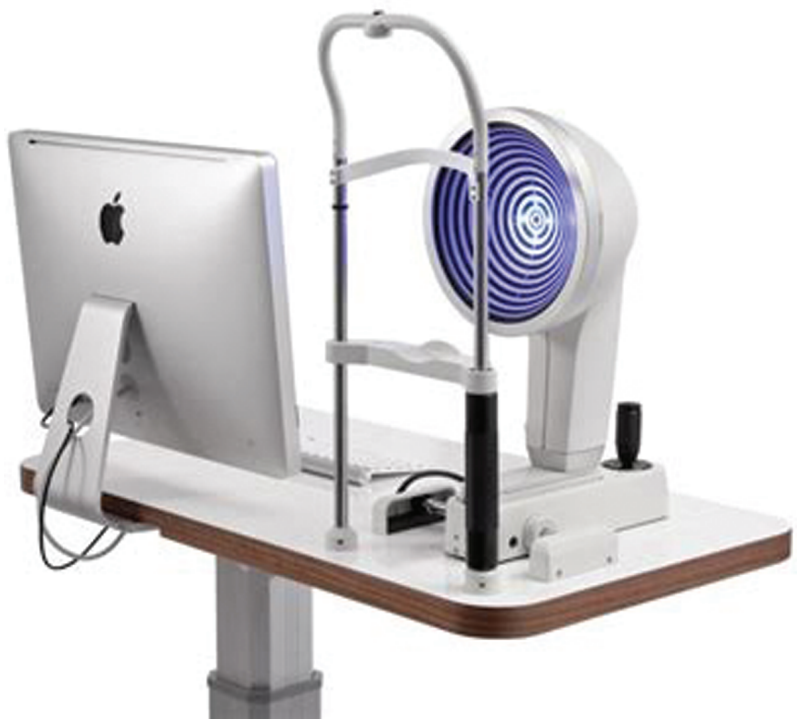

Another new device to the dry eye arena and Dry Eye University is the Quantel Medical LacryDiag. Similar to the Lipiview and the Keratography $5 \mathrm{M}$, the LacryDiag provides multiple diagnostics in one

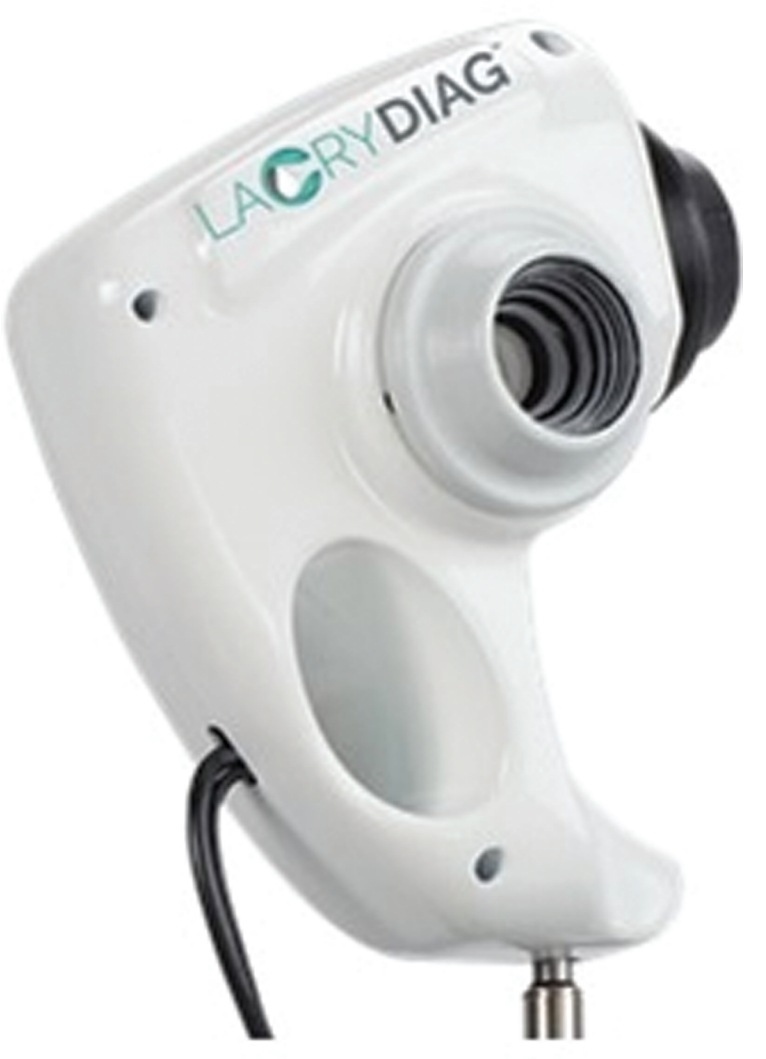

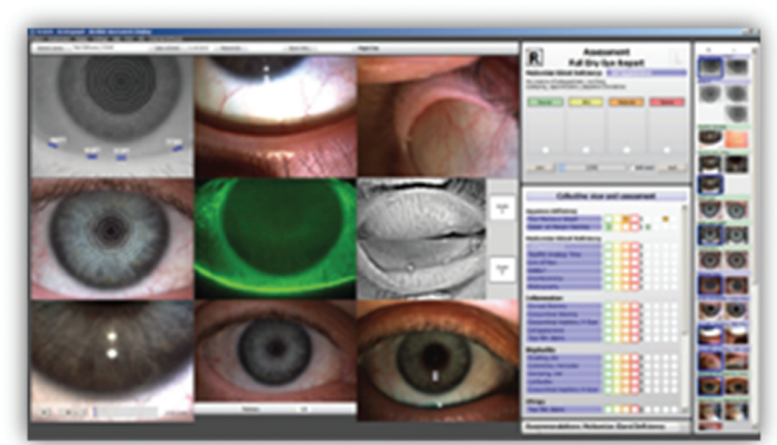

device. The LacryDiag includes four non-contact exams that can be acquired in 4 minutes. Non-invasive Tear Break Up Time, Tear Meniscus Height, Lipid Layer Interferometry and meibography are all included within its one software to provide a report of the patient's results. This device has available configurations to be slit-lamp mounted or one its own frame mount alone. It also has a travel case available to allow for easy and safe transport to multiple locations if desired.

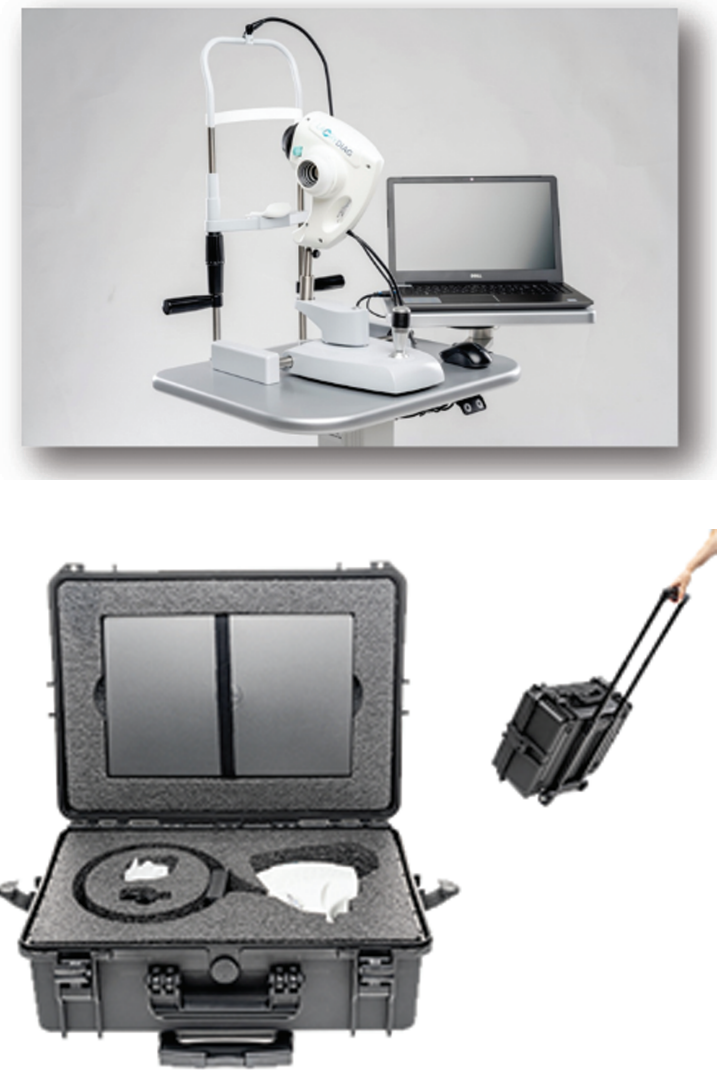

J Dry Eye Dis 3(SP1):e21-e25; April 16, 2020

This article is distributed under the terms of the Creative Commons AttributionNon Commercial 4.0 International License. (C2020 Robben. 
Bausch and Lomb has an often overlooked diagnostic available that we love at our practice. In office allergy testing using the scratch testing available through B\&L has become a welcomed addition to our testing battery. Driven by the modified SPEED, if a patient reports possible allergy symptoms we are prompted to order allergy testing. The patient returns for a testing visit done by a certified technician who performs the scratch test and results that are then reviewed with the patient at close follow up with the ordering physician. Understanding if a patient truly has environmental allergies has helped us to better guide dry eye and allergy treatments, most notably in those who have fewer allergies presented by the test results. In these cases, we can usually advise patents to discontinue allergy medications which is an advantage in patients who are also being treated for dry eye. This test is also reimbursed well through medical insurance.

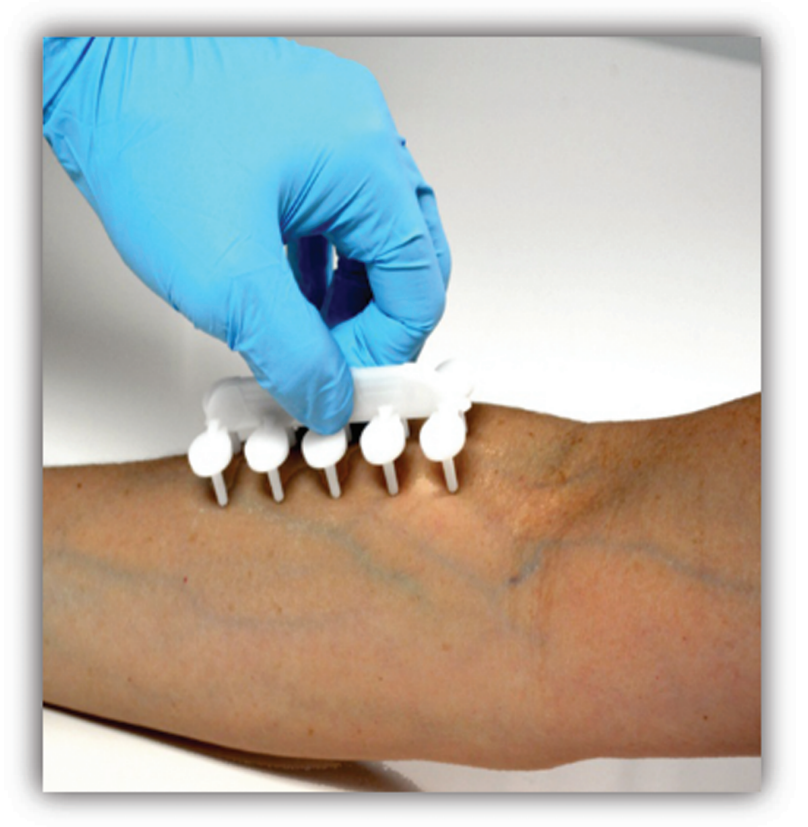

The HD Analyzer by Visiometric is another solid member of our testing battery. This device is unique in providing an objective measurement of a patients visual quality by measuring optical scatter along with aberrometry. This device assesses this scatter at the ocular surface and the lens. The information can be isolated and provided in a report that can easily show instability and fluctuation in the tear film quality and also the effects of the lens on creating poor vision. This device has proved to be invaluable in our clinic to differentiate between a patient who requires cataract surgery vs more dry eye treatments before cataract surgery and to provide them the visual evidence of these results.

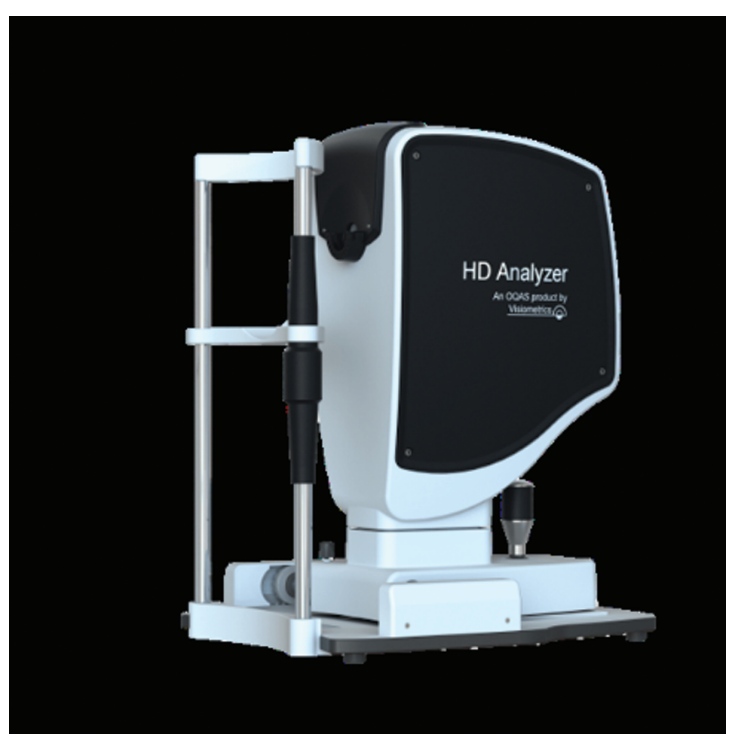

In conclusion, these diagnostics have changed our practice patterns and are a part of our standard of care for nearly every patient. Depending on what type of dry eye practice you wish to be, (referral only, shared care or full service) will determine which and how many of these diagnostics you will choose to provide in your clinic. 